\title{
Horizons/Théâtre
}

Revue d'études théâtrales

\section{2 | 2018}

Les dramaturgies arabes et l'Occident

\section{Kheireddine Lardjam : Page en construction ou quand le théâtre construit une passerelle entre deux cultures}

\section{Amel Maafa}

\section{(2) OpenEdition}

\section{Journals}

Édition électronique

URL : http://journals.openedition.org/ht/379

DOI : $10.4000 /$ ht.379

ISSN : 2678-5420

Éditeur

Presses universitaires de Bordeaux

\section{Édition imprimée}

Date de publication : 1 janvier 2018

Pagination : 98-109

ISSN : 2261-4591

\section{Référence électronique}

Amel Maafa, «Kheireddine Lardjam : Page en construction ou quand le théâtre construit une passerelle entre deux cultures », Horizons/Théâtre [En ligne], 12 | 2018, mis en ligne le 01 janvier 2019, consulté le 19 juillet 2019. URL : http://journals.openedition.org/ht/379; DOI : 10.4000/ht.379

\section{(9) $(\mathcal{\Theta \Theta}$}


Amel Maafa est maître de conférences à la faculté des Lettres et des Langues étrangères de I'Université de Guelma (Algérie). Elle enseigne les littératures francophones (maghrébine/ subsaharienne/antillaise/québécoise/Machrek) et le théâtre. Ses travaux concernent plus particulièrement l'esthétique de l'écriture maghrébine de la période post-coloniale ainsi que le théâtre algérien.

Mail: amel.maafa@gmail.com

Résumé : Kheireddine Lerdjam est un jeune metteur en scène algérien, travaillant entre l'Algérie et la France. Cette vie d'errance entre deux pays et ainsi deux cultures joue un rôle important dans ses choix de textes, voire dans ses mises en scène. Dans Page en construction, une pièce avec laquelle il a participé au Festival d'Avignon en 2015, il met en scène son propre univers, basé essentiellement sur un transfert culturel d'une rive à une autre dans un esprit de tolérance, de dialogue culturel et d'acceptation de l'Autre. La pièce de Lardjam met en scène une histoire commune qui lie deux pays, à

Abstract: Kheireddine Lerdjam is a young Algerian director, working between Algeria and France. This life of wandering between two countries, and thus two cultures, plays an important role in the choice of his texts, and in his staging. In Page en construction, a play with which he participated in the Festival d'Avignon in 2015, he stages his own universe based essentially on a cultural transfer from one bank to another in a spirit of tolerance, cultural dialogue, and acceptance of the Other. Lardjam's play depicts a common history that links two countries, through the travers I'histoire d'un homme exilé. L'auteur Fabrice Melquiot, écrit à travers le regard et la mémoire du metteur en scène, l'aventure d'un homme qui se donne, non seulement comme personne mais comme acteur, conteur et personnage. "C'est lui la page en construction». II s'agit de l'aventure de I'homme derrière l'œuvre et celle de l'œuvre derrière l'homme en mettant en avant l'essence même de l'interculturel vécu entre Français et Algériens, deux nations (dés) unies par l'h(H)istoire.

Mots-CLÉs : Théâtre- Interculturel- Histoire/ histoire- Maghreb/Occident- Mémoire

story of an exiled man. Through the eyes and memory of the director, author Fabrice Melquiot writes the adventure of a man who gives himself, not only as a person but also as an actor, storyteller and character. "He is the page under construction." It is the adventure of the man behind the work and that of the work behind the man while highlighting the essence of the intercultural lived between France and Algeria, two nations (un) united by (hi)story.

KeYwORDs: Theatre- Intercultural- History / story - Maghreb / Occident- Memory 


\section{Kheireddine Lardjam: Page en construction ou quand le théâtre construit une passerelle entre deux cultures}

JE SUIVAIS DEPUIS UN CERTAIN TEMPS DÉJÀ le travail exceptionnel d'un jeune metteur en scène algérien travaillant en France : Kheireddine Lardjam ainsi que sa dernière création Page en construction, toujours en représentation dans les villes françaises, avec laquelle il a participé au Festival d'Avignon en 2015. Cette création m’a poussée à rédiger cet article pour mettre en valeur non seulement un travail de collaboration assez atypique entre un auteur français Fabrice Melquiot et un metteur en scène algérien mais aussi le choix d'un sujet qui trouve son écho dans une actualité brûlante dans deux pays partageant plus qu'une histoire/Histoire.

Avant d'entamer mon analyse, il me semble évident de présenter l'homme derrière la scène. En plus d'être metteur en scène talentueux, Kheireddine Lardjam est aussi le directeur artistique de la compagnie «El Ajouad », crée en 1998 à Oran. Ce nom renvoie au titre d'une pièce de Abdelkader Alloula, une grande référence dans le théâtre algérien et un dramaturge déterminant dans le parcours de Lardjam, qui s'est engagé et s'engage encore à défendre son œuvre et dont il a mis en scène cinq de ses textes.

La compagnie «El Ajouad $\gg$ se consacre, comme le précise Kheireddine Lardjam lui-même', à la découverte et à la diffusion d'œuvres d'auteurs contemporains arabes tels que Noureddine Ana, Mohamed Bakhti, Rachid Boudjedra, Kateb Yacine, Tawfiq al-Hakim, Naguib Mafouz, mais aussi occidentaux, du répertoire ou contemporains.

Depuis 1999, Lardjam multiplie les collaborations en Algérie, ainsi que dans plusieurs pays arabes et en France. En 2011, il crée De la Salive comme oxygène de Pauline Sales au Théâtre de Sartrouville; en 2012, Le Poète comme boxeur de Kateb Yacine au théâtre de Béjaia, ainsi que Les Borgnes de Mustapha Benfodil à L'Arc, Scène nationale du Creusot ; en 2013, End/Igné de Mustapha Benfodil au Caire et présenté à la Manufacture lors du festival d'Avignon. En 2014, il crée Page en construction de Fabrice Melquiot à La Filature, scène nationale de Mulhouse dans le cadre du festival les «Vagamondes » en janvier 2015. La même année, il intégrera pour trois saisons l'ensemble artistique 
de la Comédie de Saint-Étienne. En mars 2016, il met en scène O-Dieux, un texte inédit de Stefano Massini sur le conflit israélo-palestinien, vu à travers les yeux de trois femmes. En 2017 Keireddine Lardjam réalisa en collaboration avec Cedric vescheembre, la pièce Saleté écrit par Robert Schneider.

Kheireddine Lardjam est l'un des rares metteurs en scène algériens dont les spectacles tournent de façon régulière en Algérie et en France.

C'est ce travail mené par Lardjam entre les deux rives de la Méditerranée qui lui a permis de se (re)trouver entre deux pays, entre deux continents, optant pour des sujets d'actualité dans un esprit de tolérance, de dialogue culturel et d'acceptation de l'Autre et de son droit à la différence.

Page en construction est avant tout une pièce qui met en place des passerelles culturelles, une création qui dépassent l'aspect local pour tendre à l'abolition des frontières qui séparent.

Quelle procédure d'écriture et de mise en scène adopter pour mettre en évidence cet aspect de la création ?

Quand Lardjam a fait une commande à Fabrice Melquiot pour cette pièce, il n’a rien exigé. L'auteur avait toute liberté dans son écriture. Le résultat était inattendu puisque l'auteur a choisi de parler du commanditaire lui-même. Melquiot a mis en place une pièce assez difficile à catégoriser où il place Lardjam au-devant de la scène en devenant le héros de sa propre histoire. Mais il ne s'agit pas d'une seule et unique histoire, ça serait ennuyeux, linéaire, prévisible. Melquiot a opté pour une multiplication d'histoires qui s'emboîtent les unes dans les autres soulignant ainsi d'autres vies d'autres personnages qui se greffent sur celle d'un homme comme pour donner de l'épaisseur à une Histoire commune liant les deux pays à travers l'histoire d'un exilé, et comme tous les exilés, «il est une terre à part entière, une île errante ${ }^{2} \gg$. L'auteur Fabrice Melquiot, écrit à travers le regard et la mémoire du metteur en scène, l'aventure d'un homme qui se donne, non seulement comme personne mais comme acteur, conteur et personnage. «C'est lui la page en construction ${ }^{3}$. » Algérien vivant en France, à moins qu'il soit Français vivant en Algérie, il vit entre les deux pays donc nulle part. Il vit le présent comme il incarne le passé. Guerre ou paix, il vit les deux dans ses souvenirs et dans ses oublis, dans sa parole et dans ses moments de silence.

Ces souvenirs sont ainsi la toile de fond de la pièce de Lardjam et du texte de Melquiot. Les deux ont tâché de ne pas réduire l'écriture théâtrale à la théâtralisation de l'écrit. Certes, le verbe au théâtre est important mais l'on ne peut «subordonner le théâtre au texte », affirmait Artaud. D’ailleurs, il disait à ce sujet : 
Un théâtre qui soumet la mise en scène à la réalisation, c'est-à-dire tout ce qu'il y a en lui de spécifiquement théâtral, au texte, est un théâtre d'idiot, de fou, d'inverti, de grammairien, d'épicier, d'anti-poète et de positiviste, c'est-à-dire d'Occidental'.

Face à « la dictature de la parole », Artaud propose « une poésie dans l'espace indépendante du langage articulé ». Face à « la suprématie de la parole $\gg$, Lardjam casse la norme occidentale. Sa mise en scène est telle que nous ne pouvons la concevoir comme limitée par le texte. Elle est un « langage propre $\gg$, un « langage théâtral pur » où il place non seulement ce qu'il a appris du théâtre occidental mais aussi toutes ses références maghreboorientales, son vécu, ses influences et ses inspirations et ses penchants artistiques. La pièce devient ainsi une œuvre trans-générique, trans-culturelle, trans-artistique.

Une entente s'est installée dès les premiers instants de la création textuelle ou représentationnelle entre l'auteur et le metteur en scène. Ce dernier fera vivre le texte mais il ira au-delà du simple fait de « réaliser une œuvre conformément à la volonté de l'auteur $\gg$, pour reprendre Édouard Bourdet ${ }^{5}$, mais tente de créer un vrai spectacle où tous les ingrédients sont présents et s'insèrent de manière à donner un sens à chaque mot mais surtout à chaque mimique, chaque chant, chaque image, chaque fait et geste sur scène.

Ceci nous mène à la première rupture avec le théâtre occidental traditionnel qui est sans nul doute le fait que la mise en scène de Lardjam est en soi « une création » pour reprendre encore une fois Artaud qui affirme que «C'est autour de la mise en scène, considérée non comme le simple degré de réfraction d'un texte sur la scène, mais comme le point de départ de toute création théâtrale [que se constituera le langage type du théâtre $\gg^{6}$. La pièce offre ainsi un spectacle non seulement fondé essentiellement sur le texte mais aussi et surtout sur le décor, les sons, les cris, la lumière et les gestes où « des images physiques violentes broient et hypnotisent la sensibilité du spectateur pris dans le théâtre comme dans un tourbillon de forces supérieures ${ }^{7} \gg \mathrm{com}^{-}$ binant dramaturgie, musique et bande dessinée, il ouvre de nouvelles perspectives à son théâtre, en réunissant tous les genres et expressions scéniques pour en faire un spectacle à part entière.

Bien évidemment, Lardjam n'a rien inventé. Appolinaire, avant lui avait imaginé un théâtre libre de tout souci de réalisme et de toute contrainte, où le travail d'écriture et de mise en scène utiliserait à loisir, « tous les mirages » mis à disposition. Dans le prologue en vers des Mamelles de Tirésias il rêvait 
d'une scène où tout peut se mélanger pour ne constituer qu'une seule entité, celle du Spectacle:

On tente ici d'infuser un esprit nouveau au théâtre

Une joie une volupté une vertu [...]

Les sons les gestes les couleurs les cris les bruits

La musique la danse l'acrobatie la poésie la peinture

Les chours les actions et les décors multiples.

$$
\text { Prologue }
$$

Lardjam a parcouru dans sa pièce tous les degrés de la hiérarchie relative entre le texte et le spectacle, en faisant de la poésie une source d'inspiration et en usant aussi bien du langage textuel que celui des gestes. Selon Ionesco ${ }^{9}$, le théâtre comporte un « langage autonome », autant « visuel » qu' « auditif », et repose sur « une architecture mouvante d'images scéniques » : « Il est donc non seulement permis, mais recommandé, de faire jouer les accessoires, faire vivre les objets, animer les décors, concrétiser les symboles ${ }^{10}$. $\gg$ Luimême entendait créer un « théâtre où l'invisible devient visible, où l'idée se fait image concrète, réalité, où le problème prend chair ${ }^{11} \gg$.

Chez Lardjam, l'image initiale du personnage debout au centre de la scène s'est greffée sur une histoire, celle d'un homme, celle des hommes qui vont l'accompagner tout au long de la pièce. Cependant, leurs histoires ne feront que renforcer l'image initiale, fondamentale qui donne sa signification à la pièce.

Un passage de la pièce pourrait résumer la démarche adoptée par l'auteur et par le metteur en scène, une citation du célèbre Scott Fitzgerald:

Avant de commencer cette brève histoire, je voudrais faire une observation d'ordre général - la marque d'une intelligence de premier plan est qu'elle est capable de se fixer sur deux idées contradictoires sans pour autant perdre la possibilité de fonctionner. On devrait par exemple pouvoir comprendre que les choses sont sans espoir, et cependant être décidé à les changer ${ }^{12}$.

Par cette citation, l'auteur met en avant le rapport contradictoire que revêt la pièce. Cette contradiction est mise au centre des préoccupations de Melquiot mais surtout de Lardjam qui en fait une source d'inspiration dans sa mise en scène. Ne vivons-nous pas dans un univers de contradictions. Page en construction ne fait que refléter notre propre image et celle du monde dans lequel nous évoluons, dans lequel nous nous mouvons pour trouver notre 
place. À travers le regard de Kheireddine Lardjam, nous nous voyons, nous nous reconnaissons. Sa mémoire est la nôtre, une mémoire qui jaillira tout au long de la pièce et mettra le spectateur face à son passé, face à son présent et à son avenir. En se dévoilant et en dévoilant sa vie, ses rêves et ses angoisses, il nous met face au mur, celui qui nous sépare, de lui, des autres, de nousmêmes.

Ceci nous rappellera peut-être une citation d'Adamov qui déclare :

J'ai compris enfin (mieux vaut tard que jamais) qu'une cuvre d'art, et surtout une pièce de théâtre, n'acquiert de réalité que si elle se place dans un contexte social défini, si sa révolution formelle se relie à une révolution interne. Je ne crois plus à cette avant-garde trompeuse qui utilise sans doute des techniques neuves mais oublie que ces techniques neuves sont nulles et non avenues si l'auteur ne se met pas au service d'une idéologie ${ }^{13}$.

Lardjam et Melquiot gardent cet esprit engagé dans leur pièce, mais l'adoptent de manière plus subtile et dans une écriture où le spectateur est amené à se poser des questions mais aussi à rire. Il est mis face à une société de plus en plus xénophobe, de plus en plus raciste mais pas dans un esprit de guérilla ou d'art de combat franc mais dans une (auto) dérision constructive vers un avenir meilleur.

L'avenir pour Lardjam se voit à travers son fils, occupé avec ses figurines de super-héros. D’ailleurs, le choix des super-héros n'est guère arbitraire. La pièce ouvre sur la scène suivante:

- Qu'est-ce que c'est, ça ? - Ça c'est Hulk. - Hulk. - Oui, c'est Hulk. - C'est qui, Hulk ? - C'est un bonhomme. - Un bonhomme vert. - Oui, il est vert. Pourquoi il est vert? - Parce qu'il est en colère. - Et qu'est-ce qui se passe, quand il est en colère ? - Il casse tout. - Il casse tout ? - Oui, tout. - Un bonhomme vert avec un short blanc et des yeux rouges. Il est aux couleurs de l'Algérie, ce Hulk. Bravo, mon fils, tu l'as bien choisi.

Dans ce passage, nous sommes face à un père discutant avec son fils en désignant la figurine d'un super-héros, Hulk. D’autres figurines apparaissent au fur et à mesure qu'on avance dans l'histoire, celle de Thor, le Dieu de l'Orage et celle de Batman, l'homme ultra-riche aux gadgets hypersophistiqués. Chaque figurine porte en elle une image. Hulk qui rappelle les couleurs du drapeau algérien, Thor et ses longs cheveux blonds que le fils rêve d'avoir, et Batman dont la richesse n'égalera pas la richesse de l'Algérie avec son pétrole et celle de la France avec ses « stations-service ». 
Le dernier super-héros n'est pas une figurine mais c'est Kheireddine Lardjam lui-même. Melquiot l'a baptisé : Algéroman.

- C'est quoi, Algéroman ? - C'est toi. - C'est moi ? - Un super-héros, un super-héros arabe. Un mec au service des gens, représentant une minorité, mais prêt à agir pour tous. Un universaliste, un mec ouvert, ce serait ça son premier superpouvoir: l'ouverture.

Ce travail d'écriture va au-delà d'une simple restitution biographique de l'auteur. Tout au long de la pièce on assiste à des bribes d'histoires et d'Histoire, à un jeu de passe-passe entre Fabrice Melquiot et Kheiredine Lardjam (la personne/le personnage). Ainsi, plutôt que d'écrire une pièce évoquant la guerre d'Algérie et la chape de silence qui recouvre l'histoire commune des deux pays, Melquiot esquive. Il fait un pas de côté et affirme qu'il composera sur le commanditaire lui-même, ce dernier se retrouve ainsi propulsé malgré lui au centre de la page.

Il est clair dès le départ : la guerre d'indépendance, il ne l'a pas faite. Il est né après. À moins qu'il ne la refasse chaque jour, comme disait Melquiot, à chaque fois qu'il parle, à chaque fois qu'il se rappelle, à chaque fois qu'il oublie, à chaque fois qu'il cite son père.

Kheireddine Lardjam endosse pour nous le costume du super-héros Algéroman cousu sur mesure par l'auteur, et nous entraîne sur un chemin à la fois poétique et fraternel. Dans un véritable emboîtement de souvenirs où s'entrecroisent plusieurs mémoires : celle de sa famille, celle des deux pays et celle du comédien-conteur, on en découvre bien davantage que dans un livre d'histoire : les souvenirs d'un père parti trop tôt, d'un appelé pris malgré lui dans une guerre qui ne le concerne pas, d'un Français qui ne voit en lui que le teint basané, d'une femme dé-voilée, etc.

Algéroman tente de nous apprendre à Tisser des liens. Il s'agit d'un véritable ressenti pour cet homme qui a pourtant l'habitude de circuler entre les deux pays depuis 15 ans. Il fait remonter à la surface les émotions de l'homme, de l'individu rattaché malgré lui à cette falaise de la Grande Histoire. La vérité c'est que la nature n'est pas figée, elle est toujours en mouvement, mais il n'est pas aisé de poser des mots sur des émotions emportées par le courant. Difficile de remuer les plaies, et justement de parler des horreurs commises par des Français pendant la guerre d'Algérie, sans créer un moment de malaise ou d'effroi.

Mon père me tire par la main jusqu'à une boulangerie. Il me dit: regarde, Kheireddine, tu vois cette boulangerie, tu la vois? Oui, je la vois. Regarde bien. Je regarde, Baba. Tu vois cette boulangerie? Oui, Baba. Est-ce que tu la vois? 
Kheireddine Lardjam : Page en construction ou quand le théâtre construit...

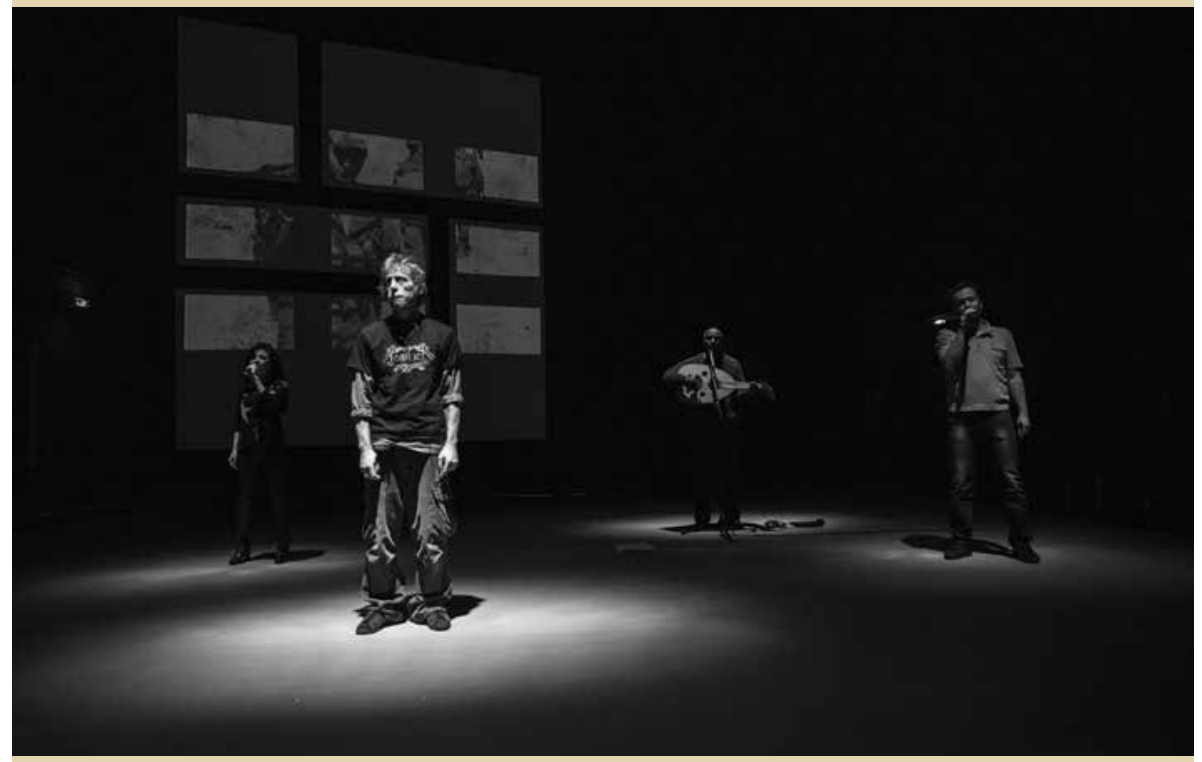

Page en construction

(Archives privées Kheirddine Lardjam)

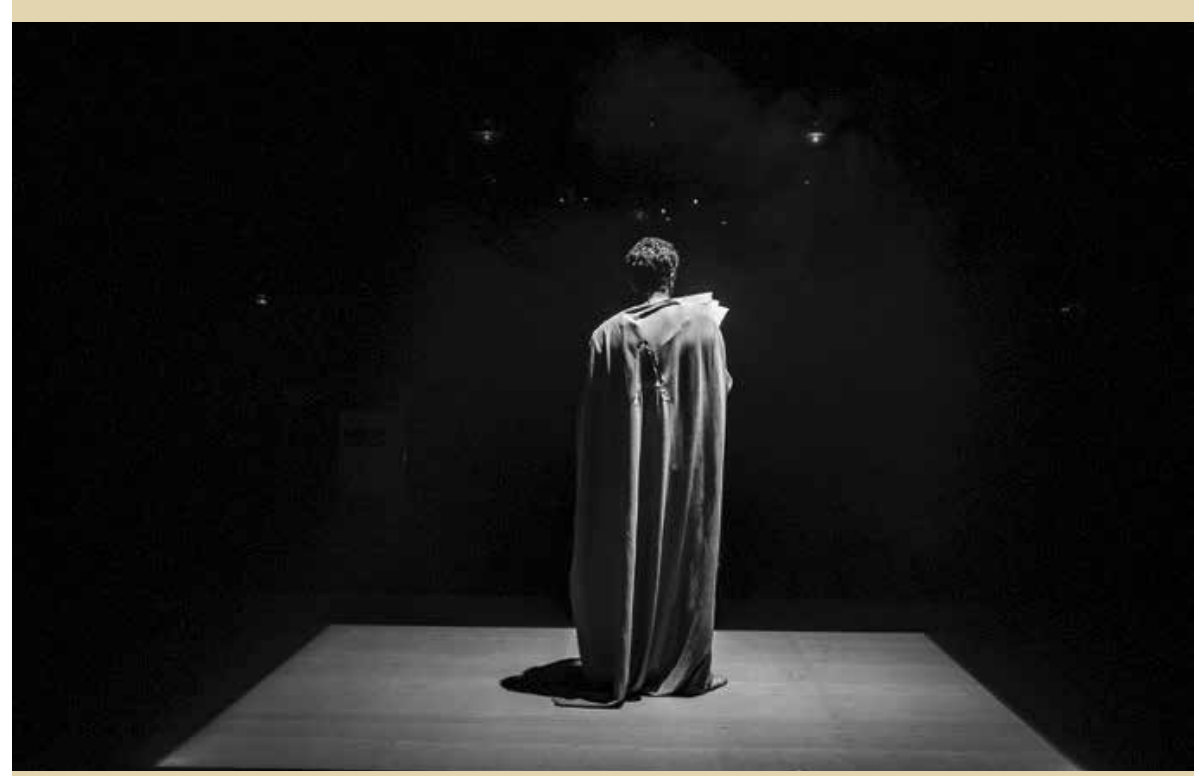

Page en construction

(Archives privées Kheirddine Lardjam) 


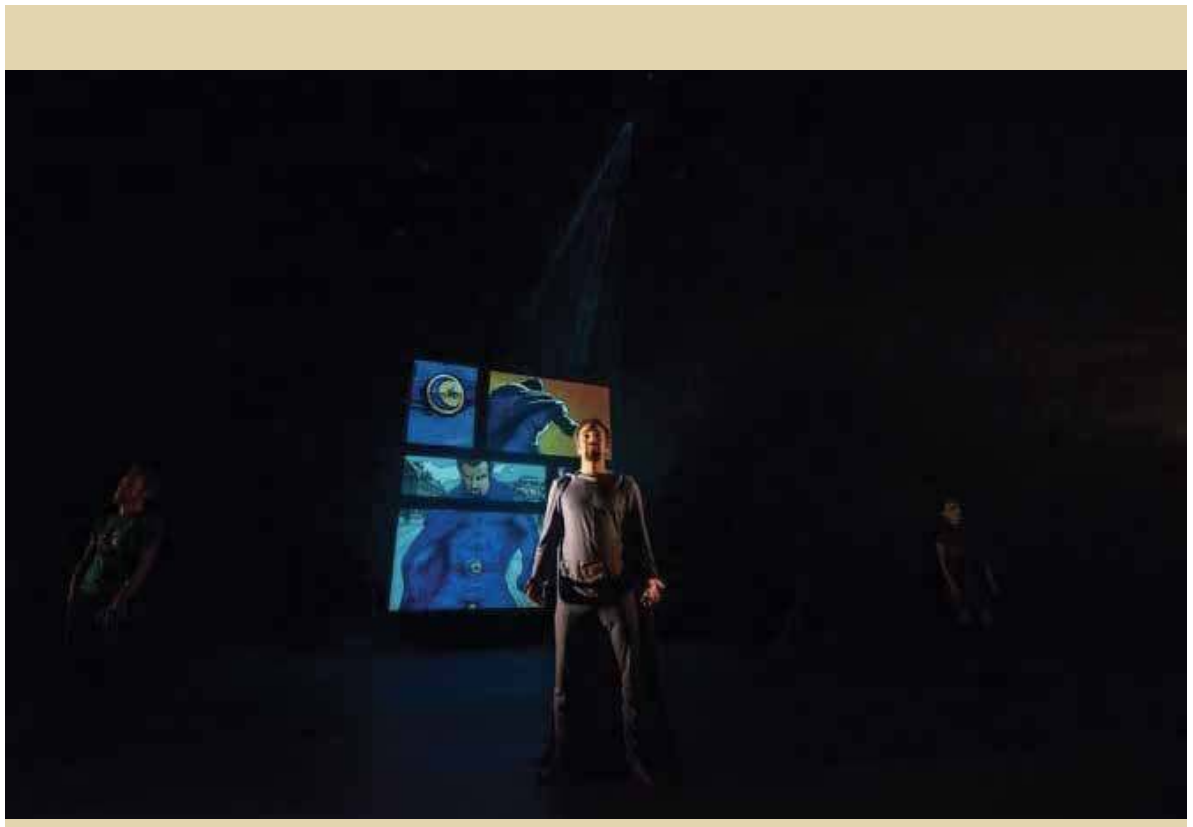

Kheirddine Lardjam dans Page en construction

(Archives privées Kheirddine Lardjam)

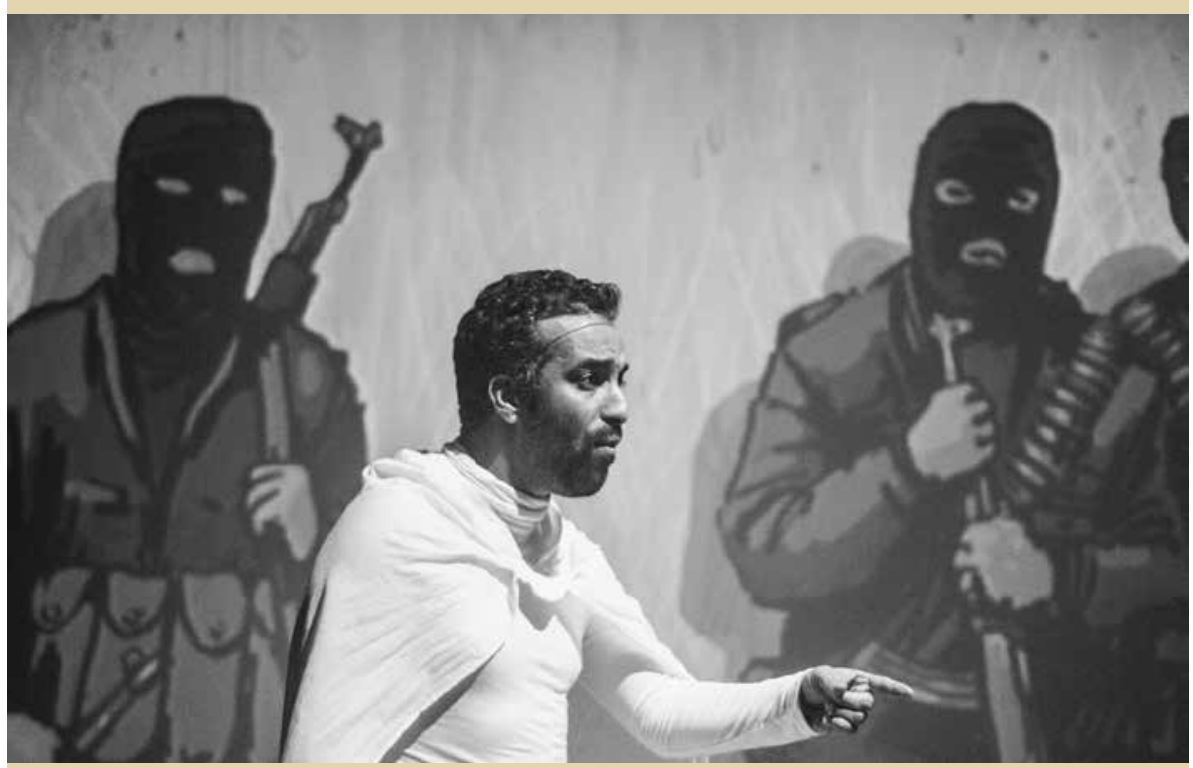

Kheirddine Lardjam dans Page en construction

(Archives privées Kheirddine Lardjam) 
Je la vois. Tu vois le four où on fait cuire le pain, tu vois le four là-bas, le four de cette boulangerie, est-ce que tu le vois Kheireddine? Je le vois, Baba, je le vois, ça sent bon, on va acheter du pain, Baba. Dans cette boulangerie, j'ai vu l'armée française brûler vive une femme algérienne, enceinte, elle attendait un bébé Kheireddine, j'ai vu les soldats français prendre la femme par le bras, la taille, la nuque, c'était des tenailles, et ils la poussaient comme on veut faire entrer une vache dans l'étable, mais c'était une femme Kheireddine, une femme algérienne, elle était enceinte, elle attendait un bébé, mon fils, ils l'ont jetée dans le four et l'ont brûlée vive. Est-ce que tu as compris? Oui, Baba. Est-ce que tu as compris ? Oui, j'ai compris. Tu es sûr ? J'ai compris, Baba. Est-ce qu'on peut acheter $d u$ pain, maintenant ${ }^{14}$ ?

Un passage relatant les atrocités de la guerre d'Algérie et mettant le spectateur face à des mots/maux crus, dans un face-à-face avec l'acteur/porteur de l'Histoire d'un pays soumis à la violence d'une colonisation et à celle de la guerre. Mais comment ne pas avoir froid au dos après ce passage ? La réponse est simple : noyer l'horreur dans l'humour et parler par exemple de son prépuce qui apparaît puis disparaît comme par enchantement ou de son sexe qui double de taille.

Parfois la mémoire et la douleur c'est comme un pendule autour du cou, ça empêche de respirer, alors les deux hommes osent l'humour, avec une mise en scène qui fait la part belle aux acteurs musiciens, Larbi Bestam et Sacha Carmen qui ont tous deux des voix magnifiques et Romaric Bourgeois, à la voix rock qui va parfaitement avec le son très éclectique de sa guitare et qui danse comme s'il lui poussait des ailes.

Il faut oser, oser, faire des déclarations d'amours à nos ennemis, nous disent encore ces deux malicieux Fabrice Melquiot et Kheireddine Lardjam. La fiction et la réalité confondues permettent de faire émerger cette Page en construction, qu'éclaire le regard généreux de toute l'équipe, généreux mais vigilant. Le regard est loin d'être manichéen. Nous n'avons pas affaire à deux espaces antithétiques, les bons d'un côté et les méchants de l'autre, mais une sorte d'appel d'amour. Même si la guerre était et est toujours meurtrière et injuste, il n'en reste pas moins qu'il ne faut pas alimenter la haine qui domine le discours politique et médiatique.

La pièce finit sur une note d'amour ou peut être d'humour et de dérision. Pour Lardjam, c'est au spectateur d'interpréter le sens et la portée d'un message sur l'actualité qui se veut inoffensif, pacifique mais combien nécessaire :

Musulman. C'est un mot qui dit je t'aime à tout le monde, sans exception. Il dit je t'aime à tous les extrémismes. Frère Musulman, je t'aime, toi l'extrémiste. Tu 
es si naïf et si violent, enfant forcené au sourcil froncé. Front National, amis du Front, Marine, je vous aime, vous qui chantez si bien le nom de mes frères de foi. Allez-y, Marine Le Pen : dites Musulman. Encore, Marine. Oh oui, encore, vous m'excitez. Musulman.

\section{Bibliographie}

Adamov Arthur, Ici et Maintenant, Paris, Gallimard, 1964.

Artaud Antonin, Le Théâtre et son double, Paris, Gallimard, 1938.

Bonnefoy Claude, Entretiens avec Eugène Ionesco, Belfond, 1966.

Lioure Michel, Lire le Théâtre moderne, Paris, Dunod, 1998.

Melquiot Fabrice, Page en construction, texte non publié.

Ryngaert Jean-Pierre, Lire le Théâtre contemporain, Paris, Dunod, 1993.

\section{Sites Web}

Apollinaire Guillaume, Les Mamelles de Tirésias: drame surréaliste en deux actes et un prologue, http://obvil.paris-sorbonne.fr/corpus/apollinaire/mamelles-detiresias/prologue (Consulté le 12/01/2017)

Ionesco Eugène, Notes et contre-notes, http://webcache.googleusercontent.com/ search?q=cache:zyPMrOzUpVEJ:lewebpedagogique.com/morinphilippe/ files/2010/01/3-Ionesco-lg-th.pdf $\& \mathrm{~cd}=1 \& \mathrm{xhl}=\mathrm{fr} \& \mathrm{ct}=\mathrm{clnk} \& \mathrm{gl}=\mathrm{pt} \quad$ (Consulté le $17 / 02 / 2017$ )

http://www.theatre-contemporain.net/spectacles/Page-en-construction/ensavoirplus/ (Consulté le 03/10/2016)

http://www.elajouad.com/fr/accueil (Consulté tout le long de la rédaction de l'article)

\section{Notes}

1. Le site de la compagnie $\mathrm{El}$ ajouad est très bien organisé et mis à jour. Lon peut trouver tout le programme des tournées, des créations de l'année en cours, voire celle de la saison prochaine. http://www.elajouad.com (Dernière consultation le 13/05/2017)

2. http://www.theatre-contemporain.net/spectacles/Page-en-construction/ensavoirplus/ (Consulté le 03/10/2016)

3. http://www.elajouad.com/fr/actualites/page-en-construction (Consultéle 10/10/2016)

4. Antonin Artaud, Le Théâtre et son double, Paris, Gallimard, 1938 in Jean-Pierre Ryngaert, Lire le Théâtre contemporain de Dunod, Paris, 1993, p. 20.

5. Lettre à Edouard Bourdet, 31 juillet 1939 in J.P. Ryngaert Lire le Théâtre contemporain, op. cit., p. 23. 
Kheireddine Lardjam : Page en construction ou quand le théâtre construit...

6. A. Artaud, Le Théâtre et son double, op. cit. in J.P. Ryngaert Lire le Théâtre contemporain, op. cit., p. 24.

7. Ibid., p. 24.

8. Guillaume Apollinaire, Les Mamelles de Tirésias: drame surréaliste en deux actes et un prologue, http://obvil.paris-sorbonne.fr/corpus/apollinaire/mamelles-de-tiresias/ prologue (Consulté le 12/01/2017).

9. Eugène Ionesco, Notes et contre-notes, http://webcache.googleusercontent. $\mathrm{com} /$ search?q=cache:zyPMrOzUpVEJ:lewebpedagogique.com/morinphilippe/ files/2010/01/3-Ionesco-lg-th.pdf+\&cd=1\&hl=fr\&ct=clnk\&gl=pt (Consulté le 17/02/2017).

10. Ibid.

11. Ibid.

12. Citation tirée du texte de F. Melquiot.

13. Arthur Adamov, Ici et Maintenant, Gallimard, 1964, in J.P. Ryngaert, Lire le Théâtre contemporain, op. cit., p. 35.

14. Citation tirée des pages 19 et 20 du texte de Melquiot. 